\title{
Transport of exogenous surfactants on a thin viscous film within an axisymmetric airway
}

\author{
Wu-ting Tsai ${ }^{\text {a,b,*, }}$ Li-Yu Liu ${ }^{b}$ \\ ${ }^{a}$ Institute of Hydrological Sciences, National Central University, Jungli, Taoyuan 320, Taiwan \\ ${ }^{\mathrm{b}}$ Department of Civil Engineering, National Chiao Tung University, Hsinchu 300, Taiwan
}

Received 10 July 2003; accepted 10 December 2003

\begin{abstract}
The transport of exogenous surfactant in an axisymmetric pulmonary airway lining is analyzed using the model based on lubrication theory to understand the parameters involved in surfactant replacement therapy for neonatal respiratory distress syndrome, including the ratio of film thickness-to-airway radius $\varepsilon$, and the initial exogenous and endogenous surfactant distributions. Numerical simulation of the model indicates that the film disturbance induced by Marangoni flow in low generation airways (small $\varepsilon$, such as trachea) would be more significant than that in high generation airways (large $\varepsilon$, such as bronchioles) for the same amount of instilled surfactant. The non-dimensional transport properties of the exogenous surfactant, however, are virtually identical in various airway generations considered. Increasing the initial local exogenous concentration enhances the surfactant transport and also intensifies the film disturbance. The presence of endogenous surfactant tends to moderate the shock-like film disturbance and also prolongs the extent of surfactant monolayer front. This, however, does not mean that the spread of exogenous surfactant is enhanced with the presence of endogenous surfactant. The extended monolayer is attributed to the compression of endogenous surfactant concentration in regions ahead of the advancing exogenous surfactant front.
\end{abstract}

(C) 2003 Elsevier B.V. All rights reserved.

Keywords: Surfactant; Pulmonary airway; Thin film; Lubrication theory; Surfactant replacement therapy

\section{Introduction}

The interiors of pulmonary airways and alveoli are covered with a thin layer of viscous liquid lining. The pulmonary surfactant, secreted by type II epithelia cells in the alveolar zone, lowers the surface tension of the mucous film to not only increase lung compliance but also make the breathing smooth. With insufficient or inactive surfactant, the lungs will not expand easily and breathing becomes difficult. Neonatal respiratory distress syndrome (termed RDS) occurs in prematurely born infants when type II epithelia cells do not mature to produce adequate quantities of pulmonary surfactant [1]. Infants suffering from RDS breathe strenuously and may ultimately develop exhaustion, alveoli collapse, and suffocation due to low lung compliance.

Fujiwara et al. [2] were among the pioneering attempts to treat neonatal RDS by endotracheal administration of arti-

\footnotetext{
${ }^{*}$ Corresponding author. Tel.: +886-3-34271052; fax: +886-3-34222894

E-mail address: wttsai@cc.ncu.edu.tw (W.-t. Tsai).
}

ficial surfactants derived from animal lungs. Surfactant replacement therapy (termed SRT) is now the standard treatment for infants suffering from RDS [3]. The procedure involves instilling the exogenous surfactant dose into the trachea via an endotracheal tube. The neonate is then ventilated for a short period before the next dose is instilled. When the surfactant is introduced into the trachea, it lowers the local surface tension and forms surface tension gradient between the trachea and other smaller airways. The induced surface tension gradient results in a flow, called Marangoni flow, in the direction towards the higher surface tension (lower surfactant concentration) regions [4]. Through the Marangoni effect, the exogenous surfactants are delivered to the peripheries of surfactant-deficient alveoli. Despite the success of SRT coupled with mechanical ventilation strategies, RDS still remains the fourth leading cause of death among premature neonates in the US [5]. Furthermore, approximately $30 \%$ of those treated by SRT show only a transient response or do not respond at all to the treatments [6].

Being provoked by the need to improve techniques and efficiency for SRT, the mechanisms of surface tension-driven 
interfacial flows within the pulmonary airways have recently been the subject of active research (see the recent review by Grotberg [7]). Examples of these studies include those by Grotberg and co-workers [8-13], who explored systematically the transport of surfactants on a planar, thin, viscous film under various conditions and the parameters which control the processes, and the work by Davis et al. [14], Espinosa [15], Espinosa et al. [16], who studied the spreading of surfactants in an axisymmetric, film-lined airway. By assuming a thin lined film, these studies employed the lubrication theory to analyze the transport of surfactants and the deformation of airway lining. The ultimate objectives of these theoretical models are to obtain a better estimate of the transit time needed for the surfactants to reach the lungs, and also to improve the efficiency of SRT through understanding of the detailed transport mechanisms. Nonetheless, given the complexity of the pulmonary airways and the vast variety of parameters that can influence the underlying physics, contradictory results of the transit time for the surfactants to reach the periphery were predicted from separate models. This is primarily attributed to the parameters involved and the treatments of real airway geometry that have profound effect on the behavior of the model. In this work, we reappraise the problem of surfactant spreading in an airway lining with the emphases on clarifying the underlying mechanisms that govern the delivery of exogenous surfactants and on assessing the possible impact of the surfactant redistribution on the liquid lining in surfactant replacement therapy.

\section{Lubrication model}

We focus on localized transport of an exogenous surfactant within a single airway lined with a thin liquid layer as considered in Davis et al. [14] and Espinosa et al. [16]. The transport model to be investigated is similar to that in Espinosa et al. [16], and a brief summary of the formulation is given in the following.

An axisymmetric airway of circular cross-section with radius $\tilde{R}$ and length $\tilde{L}$ is considered. A schematic of the model geometry and the cylindrical coordinate system is shown in Fig. 1 . The $r$-axis points to the airway radial direction, and the $z$ coordinate coincides with the airway centerline. Initially, the interior of the airway is covered with an undisturbed, thin layer of liquid lining with a uniform thickness



Fig. 1. The geometry of a lined airway with insoluble surfactants distributed. $\tilde{h}_{0}$. The liquid lining is assumed to be incompressible with a constant density $\tilde{\rho}$ and Newtonian with a constant viscosity $\tilde{\mu}$.

At the air-liquid interface of the lining, the surface tension is $\tilde{\sigma}_{0}$ when no surfactant is present. The presence of insoluble surfactant forms a monolayer that is redistributed by the advection of the liquid lining and the diffusion of the surfactant itself. The surface tension at the air-liquid interface decreases upon increasing the local surfactant concentration. The relationship between the surface tension and the surfactant concentration, which is called the equation of state, is in general non-linear and empirically determined $[17,18]$. To encompass a large range of surfactant concentration a piecewise equation of state is required. Curve fitting of the experimental measurements for surfactant concentration below the saturated limit reveals matching of a non-linear Frumkin equation of state for the high surface tension region with a linear equation for the low surface tension regime [18]. For the model problem considered in the present study (see next section for the initial condition), the initial surfactant distribution spreads quickly after the instillation causing immediate reduction in the surface tension (see, e.g. Fig. 2). The linear equation of state is applicable except for region near the spreading front of the surfactant monolayer where the surface tension is high. Previous studies [8,9] using non-linear equation of state indicate that only the disturbance of lining might be affected by the equation of state. The transport of surfactant, however, is only slightly dependent on the choice of the equation of state. Linear functional relationship is therefore used in this study.

By use of the surfactant-free surface tension $\tilde{\sigma}_{0}$ to scale the surface tension, the non-dimensional linear equation of state is

$\sigma=1-\alpha \Gamma$

where $\Gamma=\Gamma(z, t)$ is the non-dimensional surfactant concentration scaled by the saturated concentration $\tilde{\Gamma}^{*}$. The constant $\alpha=\left(\tilde{\sigma}_{0}-\tilde{\sigma}^{*}\right) / \tilde{\sigma}_{0}$ depends on the minimum surface tension $\tilde{\sigma}^{*}$ at the saturated surfactant concentration. For typical lung surfactant, $\tilde{\sigma}_{0} \approx 70 \mathrm{dyn} / \mathrm{cm}$ and $\tilde{\sigma}^{*} \approx 25 \mathrm{dyn} / \mathrm{cm}$ $[18,19]$, which results in $\alpha \approx 0.64$. The non-dimensional concentration $\Gamma$ ranges from 0 to 1 with the corresponding non-dimensional surface tension decreases from 1 to $\tilde{\sigma}^{*} / \tilde{\sigma}_{0}$.

By assuming the condition that $\varepsilon=\tilde{h}_{0} / \tilde{R} \ll 1$, lubrication theory can then be used, where $\varepsilon$ is called the lubrication parameter. The variables of the geometry and the flow are rescaled by the corresponding characteristic scales such that the non-dimensionalized variables are all of order $\mathrm{O}(1)$. The spatial derivatives with respect to $z$ and $r$ are therefore rescaled by $\tilde{R}$ and $\tilde{h}_{0}$, respectively. Solenoidal condition of the substrate flow requires that the radial velocity be $\mathrm{O}(\varepsilon)$ of the axial velocity. The characteristic axial velocity $\tilde{\sigma}_{0} \varepsilon / \tilde{\mu}$ can be considered as the shear velocity of the viscous layer 
driven by a characteristic surface tension. Accordingly, if we choose to scale the axial velocity by $\tilde{\sigma}_{0} \varepsilon / \tilde{\mu}$, the radial velocity will be scaled by $\tilde{\sigma}_{0} \varepsilon^{2} / \tilde{\mu}$. The non-dimensional radial and axial velocities, $v(r, z, t)$ and $w(r, z, t)$, are of order $\mathrm{O}(1)$. The non-dimensional thickness of liquid film $h(z, t)$, which is scaled by the undisturbed thickness $\tilde{h}_{0}$, is also of order $\mathrm{O}(1)$.

For the airways considered in this study, the Reynolds number is $R e=\tilde{\rho} \tilde{\sigma}_{0} \tilde{h}_{0} \varepsilon^{2} / \tilde{\mu}^{2} \ll 1$. In the non-dimensional radial momentum equation, the inertial terms and the viscous terms are of order $\mathrm{O}(\varepsilon R e)$ and $\mathrm{O}(\varepsilon)$, respectively. Retaining only the leading-order radial gradient of pressure term and neglecting other higher-order terms, the radial momentum equation implies that $p=p(z, t)$. For the axial momentum equation, neglecting the higher-order inertial terms of $\mathrm{O}(R e)$, and the viscous term of $\mathrm{O}\left(\varepsilon^{2}\right)$, it becomes

$0=-\varepsilon p_{z}+r^{-1}\left(r w_{r}\right)_{r}$.

At the air-liquid interface, $\varepsilon r=1-\varepsilon h(z, t)=\eta(z, t)$, balance of the tangential shear stresses gives rise to the boundary condition, to leading order, as

$w_{r}=\sigma_{z}$.

Integrating the axial momentum equation (2) and imposing the interfacial tangential-stress condition (3) and the no-slip condition at the airway wall, the axial velocity can then be obtained as [14]

$\varepsilon w(r, z, t)=-\frac{1}{4} p_{z}\left[1-\eta^{2}+2 \eta^{2} \ln \eta\right]-\eta \sigma_{z} \ln \eta$.

In the normal direction of the air-liquid interface, surface tension induces normal stresses attributed to circumferential and interfacial curvatures, and are balanced by the pressure. To leading order, the normal stress condition at the interface is

$p(z, t)=\sigma\left(-\kappa \eta^{-1}+\kappa^{3} \eta_{z z}\right)$,

where $\kappa=\left[1+\left(\varepsilon h_{z}\right)^{2}\right]^{-1 / 2}$.

In addition to momentum conservation, the substrate flow should also satisfy the conservation of mass. Balancing the change in the rate of mass and the influx/outflux in a control volume, the mass conservation equation can be expressed as

$h_{t}+\eta^{-1} q_{z}=0$,

where the flux $q$ through a cross-sectional area with thickness $h$ is

$$
\begin{aligned}
q(z, t)= & -\frac{1}{4} \varepsilon^{-2} p_{z}\left[\frac{1}{4}\left(1-\eta^{2}\right)\left(1-3 \eta^{2}\right)-\eta^{4} \ln \eta\right] \\
& +\varepsilon^{-2} \eta \sigma_{z}\left[\frac{1}{4}\left(1-\eta^{2}\right)+\frac{1}{2} \eta^{2} \ln \eta\right] .
\end{aligned}
$$

On the interface, the distribution of the surfactant concentration $\Gamma=\Gamma(z, t)$ is governed by the convection-diffusion equation. For monolayer diffusion in the typical airway lining of human lungs ( $\tilde{\sigma}_{0} \approx 70 \mathrm{dyn} / \mathrm{cm}, \tilde{\mu} \approx 10^{-2} \mathrm{dyn} \mathrm{s} / \mathrm{cm}^{2}$, $\left.\tilde{h}_{0} \approx 10^{-3} \mathrm{~cm}\right)[20,21]$, the surface diffusivity $\tilde{D}_{\mathrm{s}} \approx$ $10^{-5} \mathrm{~cm}^{2} / \mathrm{s}[22]$ and the Pelect number, $P e=\tilde{h}_{0} \tilde{\sigma}_{0} / \tilde{\mu} \tilde{D}_{\mathrm{s}}$, which characterizes the convection-diffusion transport ratio, is of order $\mathrm{O}\left(10^{5}\right)$. This means that the surface diffusion of surfactant is negligible in comparison with the convection. Expressing the surfactant concentration $\Gamma(z, t)$ in terms of the new parameter $\beta(z, t)=\Gamma \eta \kappa^{-1}$, the surfactant transport equation can be expressed as

$\beta_{t}+\partial_{z}\left(\beta[w]_{r=\eta}\right)=0$,

where $[w]_{r=\eta}$ is the axial velocity at the air-liquid surface.

\section{Numerical solutions}

The leading-order transport equations (6) and (8) govern the evolution of the film thickness $h(z, t)$ and the distribution of the concentration parameter $\beta(z, t)$ with axial velocity $w(z, t)$ and pressure $p(z, t)$ given by Eqs. (4) and (5). The coupled non-linear partial differential equations are solved numerically. Third-order Adams-Bashforth scheme is used for time integrations. The spatial derivatives are approximated by second-order central difference scheme.

In the numerical results presented below, the length of the airway is assumed to be eight times of the radius, and accordingly, the non-dimensional axial coordinate ranges from $z=0$ to 8 . The initial non-dimensional thickness of the film is assumed to be uniform such that $h(z, 0)=1$. To simulate the transport of an instilled surfactant dose, the initial non-dimensional surfactant distribution is prescribed by $\Gamma(z, 0)=\Gamma_{\text {end }}+\left[\Gamma_{\text {exo }}-\Gamma_{\text {end }}\right] \cos ^{2}(1 / 2 \pi z)$ for $0 \leq z \leq$ $L_{\mathrm{exo}}$, and $\Gamma(z, 0)=\Gamma_{\text {end }}$ otherwise, which represents localized concentration around the entrance of the airway $z=0$. The initial extent of the exogenous surfactant distribution is assumed to be $L_{\text {exo }}=1$ with the maximum exogenous concentration $\Gamma_{\text {exo }}$ occurs at $z=0$. The pre-existing endogenous surfactant concentration $\Gamma_{\text {end }}$ is assumed to be uniform at the entire lining.

With the prescribed initial surfactant distribution, the concentration is symmetric about $z=0$. The induced Marangoni flow is also symmetric about $z=0$ and accordingly the axial velocity $w$, the substrate flux $q$ and the gradient of lining thickness $h_{z}$ all vanish at the entrance of the airway. From (4), (5) and (7), this implies that $\sigma_{z}$, $p_{z}$ and $h_{z z z}$ also need to be zero at the entrance. The same boundary conditions are imposed at the end of the airway.

\section{Results and discussion}

\subsection{Film disturbance and surfactant spread in various generations of airways}

The pulmonary airways in anatomy are characterized by 23 generations of branching from the trachea (generation 0 ) to the alveoli (generation 23) [23]. For the first 17 generations, the branch radius $\tilde{R}$ ranges from $9 \mathrm{~mm}$ of trachea to $0.23 \mathrm{~mm}$ of respiratory bronchiole, the length $\tilde{L}$ from 120 
to $1.2 \mathrm{~mm}$, and the ratio of film thickness-to-airway radius $\varepsilon=\tilde{h}_{0} / \tilde{R}$ from 0.001 to 0.043 . The thickness $\tilde{h}_{0}$ of the liquid film adhering to the inner side of the airway wall is approximately $10 \mu \mathrm{m}$ for all branching [20]. To exemplify the transports of surfactants on various generations of airways, four representative ratios of film thickness-to-airway radius, $\varepsilon=0.001,0.004,0.01$ and 0.043 , corresponding to generations 0 (trachea), 3 (beginning of bronchiole), 7, and 17 (terminal bronchiole) are computed. The endogenous surfactant concentration $\Gamma_{\text {end }}$ is assumed to vanish and the maximum exogenous concentration reaches the saturated limit, $\Gamma_{\text {exo }}=1$.

The time varying profiles of the non-dimensional film thickness $h(z, t)$ and the surfactant concentration $\Gamma(z, t)$ for


Fig. 2. Time varying profiles of film thickness $h(z, t)$ and surfactant concentration $\Gamma(z, t)$ for the ratio of film thickness-to-airway radius $\varepsilon=0.001$ (a, e), $0.004(\mathrm{~b}, \mathrm{f}), 0.01(\mathrm{c}, \mathrm{g})$ and $0.043(\mathrm{~d}, \mathrm{~h})$. The endogenous surfactant concentration is assumed to vanish, $\Gamma_{\text {end }}=0$. 



Fig. 3. Temporal evolutions of (a) the peak film thickness $h_{\text {peak }}$, (b) the axial coordinate of the peak $z_{\text {peak }}$, (c) the trough film thickness $h_{\text {trough }}$ and (d) the film thickness at entrance $h(0, t)$ for different values of ratio of film thickness-to-airway radius $\varepsilon=0.001,0.004,0.01$ and 0.043 .

To further reveal the effect of the ratio of film thicknessto-airway radius $\varepsilon$ on the film disturbance induced by Marangoni flow, temporal evolutions of (a) the peak film thickness $h_{\text {peak }}$, (b) the axial coordinate of the peak $z_{\text {peak }}$, (c) the trough film thickness $h_{\text {trough }}$ and (d) the film thickness at entrance $h(0, t)$ are shown in Fig. 3. As depicted in Fig. 2, as soon as the surfactant begins to spread, a shock-like solitary wave develops immediately with its peak thickness $h_{\text {peak }}$ increases drastically to more than one and a half of the initial film thickness (Fig. 3(a)). As time proceeds, the peak thickness slowly approaches an asymptotic value. The undisturbed thickness $\tilde{h}_{0}$ remains approximately to be constant $\left(\approx 10^{-3} \mathrm{~cm}\right)$ in different generations of airways $[20,21]$. The dimensional asymptotic peak thickness, therefore, is higher for smaller $\varepsilon$ (i.e. larger radius) airway, indicating that the disturbance induced by Marangoni flow in a trachea would be more significant than that in bronchioles for the same amount of instilled surfactant. Such a solitary disturbance wave propagates with the same non-dimensional forward speed for different generations of airways as indicated by the change in axial position of the peak thickness plotted in Fig. 3(b). Accordingly, this implies that the film disturbance would travel faster in a higher-generation (larger $\varepsilon$ ) airway.

Accompanying the rise of peak film height, the trough thickness $h_{\text {trough }}$ behind the solitary wave reduces as shown in Fig. 3(c). Again, such a depressed disturbance occurs more significantly in lower-generation (smaller $\varepsilon$ ) airways.
The non-dimensional decreasing rate of film thickness at the entrance of airway, $h(0, t)$, however, is independent of $\varepsilon$ (Fig. 3(d)). Film rapture occurs when the depressed trough thickness $h_{\text {trough }}$ continues to decrease as the shock-like disturbed wave propagates forward. Such a rupture in airway lining ceases the transport of surfactant [10] and should be avoided in clinical treatment. Although the film will eventually reconnect, the treatment, however, is delayed. In order to study the possibility of film rapture occurrence, in particular in the lower-generation airways where significant film disturbances are induced, simulation for trachea $(\varepsilon=0.001)$ is re-examined by extending the non-dimensional length of airway to 20 (the typical non-dimensional length of trachea is about 13). The subsequent developments of the film thickness $h(z, t)$ and the change of the trough thickness $h_{\text {trough }}$ are shown in Fig. 4. Though the trough height continues to decrease, it tends to approach an asymptotic thickness as time proceeds (Fig. 4(b)). As the front of the disturbance wave reaches the end of the airway $(z \approx 13$ at $t \approx 400$ ), the trough height $h_{\text {trough }}$ decreases to approximately $20 \%$ of the initial thickness. Film rupture never occurs as the disturbance wave travels across the entire extended airway.

In comparison with the film disturbance, which is different on various generations of airway lining, the non-dimensional properties of surfactant transport are virtually unaffected as shown in Figs. 2(e)-(h) and 5 where temporal evolutions of transport properties are plotted. In Fig. 5, the length of 



Fig. 4. Time varying profiles of film thickness $h(z, t)$ within an extended airway and $\varepsilon=0.001$ (a) and the temporal evolution of the trough film thickness $h_{\text {trough }}(\mathrm{b})$.

monolayer $L_{\text {mono }}$ (a) is defined by the position of surfactant front where the local concentration is $1 \%$ of the initial maximum concentration, and the spreading velocity of surfactant $\mathrm{d} L_{\text {mono }} / \mathrm{d} t(\mathrm{~b})$ is obtained by time differentiating the frontal position the monolayer. At the moment when the surfactant is instilled, the spreading velocity is the maximum. The velocity quickly decelerates to a smaller level while the length of monolayer increases. The initial spread of surfactant distribution results in an abrupt drop of the concentration at the entrance of airway $\Gamma(0, t)$ as shown in Fig. 5(c). Similar to the surfactant-front advancing velocity $\mathrm{d} L_{\text {mono }} / \mathrm{d} t$, the dropping rate $\mathrm{d} \Gamma(0, t) / \mathrm{d} t$ decreases quickly from an extremely high speed initially (Fig. 5(d)). The most drastic feature is that these non-dimensional properties of surfactant transport are independent of the ratio of film thickness-to-airway radius.

\subsection{The effect of the initial exogenous surfactant strength}

Whether stronger surfactant dose will result in better curative effect is critical in clinical therapy due to high cost of SRT. To study the effect of the initial exogenous surfactant strength on surfactant transport, various initial exogenous surfactant concentrations, $\Gamma_{\text {exo }}=1,0.8,0.6$ and 0.4 are used in the simulations for generation 7 airway $(\varepsilon=0.01)$. The endogenous surfactant is assumed to vanish. Fig. 6 shows the temporal developments of film disturbances and the spread of exogenous surfactant for the four values of $\Gamma_{\text {exo }}$. As the result indicates, reducing the exogenous strength decreases the initial Marangoni stress and consequently slows down the spread of surfactant and the propagation of film disturbance wave. The evolutions of the non-dimensional properties of film disturbance shown in Fig. 7 also evidence the impact of exogenous surfactant strength on film disturbance. Accompanying the acceleration of monolayer spread, the rise of the peak film height $h_{\text {peak }}$, the depression of the trough film thickness $h_{\text {trough }}$ and also the film thickness at entrance $h(0, t)$ are all intensified. Although the higher strength


Fig. 5. Temporal evolutions of (a) the length of monolayer $L_{\text {mono }}$, (b) the spreading velocity of surfactant $\mathrm{d} L_{\text {mono }} / \mathrm{d} t$, (c) the concentration at the entrance of airway $\Gamma(0, t)$ and (d) the dropping rate $\mathrm{d} \Gamma(0, t) / \mathrm{d} t$ for different values of ratio of film thickness-to-airway radius $\varepsilon=0.001,0.004,0.01$ and 0.043 . 

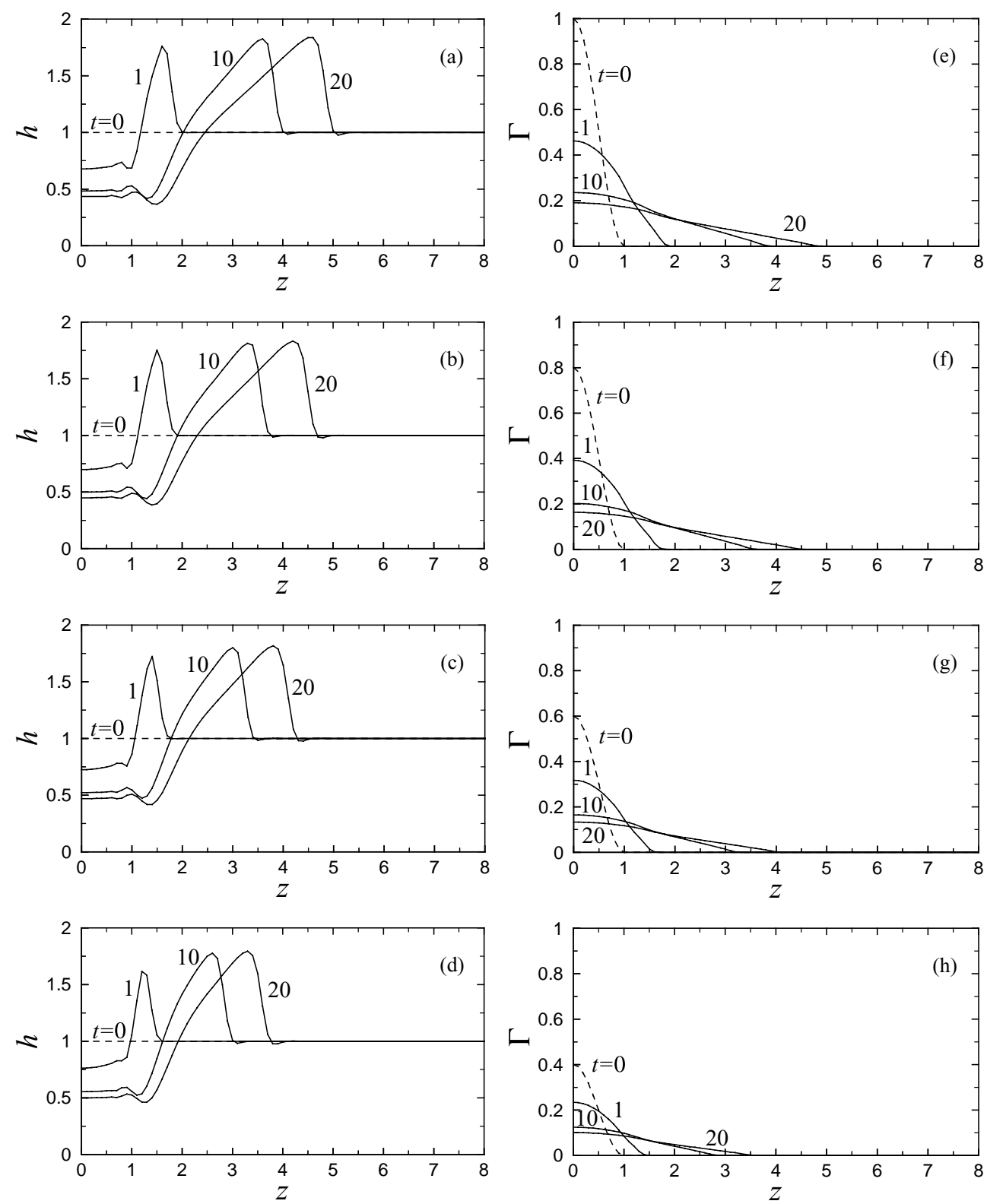

Fig. 6. Time varying profiles of film thickness $h(z, t)$ and surfactant concentration $\Gamma(z, t)$ for the exogenous surfactant concentration $\Gamma_{\text {exo }}=1(\mathrm{a}$, e), 0.8 $(\mathrm{b}, \mathrm{f}), 0.6(\mathrm{c}, \mathrm{g})$ and $0.4(\mathrm{~d}, \mathrm{~h})$. The endogenous surfactant concentration is assumed to vanish, $\Gamma_{\text {end }}=0$, and the ratio of film thickness-to-airway radius $\varepsilon=0.01$.

of exogenous surfactant enhances the monolayer spreading initially, the surfactant-front advancing velocity $\mathrm{d} L_{\text {mono }} / \mathrm{d} t$ and the dropping rate at the entrance $\mathrm{d} \Gamma(0, t) / \mathrm{d} t$ quickly decrease to similar levels as that of weaker strength initial concentration. In addition, increasing the strength of the exogenous surfactant does not necessarily proportionally improve the efficiency of monolayer spread as revealed by the increase of the monolayer length $L_{\text {mono }}$ in Fig. 8.

\subsection{The effect of endogenous surfactants}

In all cases discussed herein, resident surfactants are assumed to be absent in the airway lining. In reality, pre- existing surfactants from previous instillation or the endogenous surfactants can further complicate the transport. Such an issue has been addressed previously $[13,16,24]$. Here we consider four endogenous surfactant concentrations, $\Gamma_{\mathrm{end}}=$ $0,0.01,0.05$, and 0.1 , which distribute uniformly in the entire lining initially. The developments of the film disturbance and the surfactant spread are illustrated in Fig. 9 and the temporal evolutions of the non-dimensional film height and concentration properties are plotted in Figs. 10 and 11. As what has been observed in previous studies [13,24], the existence of the resident surfactant moderates the shock-like front of the disturbed film, even for the low endogenous-surfactant concentration considered (Fig. 9). Both the peak height $h_{\text {peak }}$ 

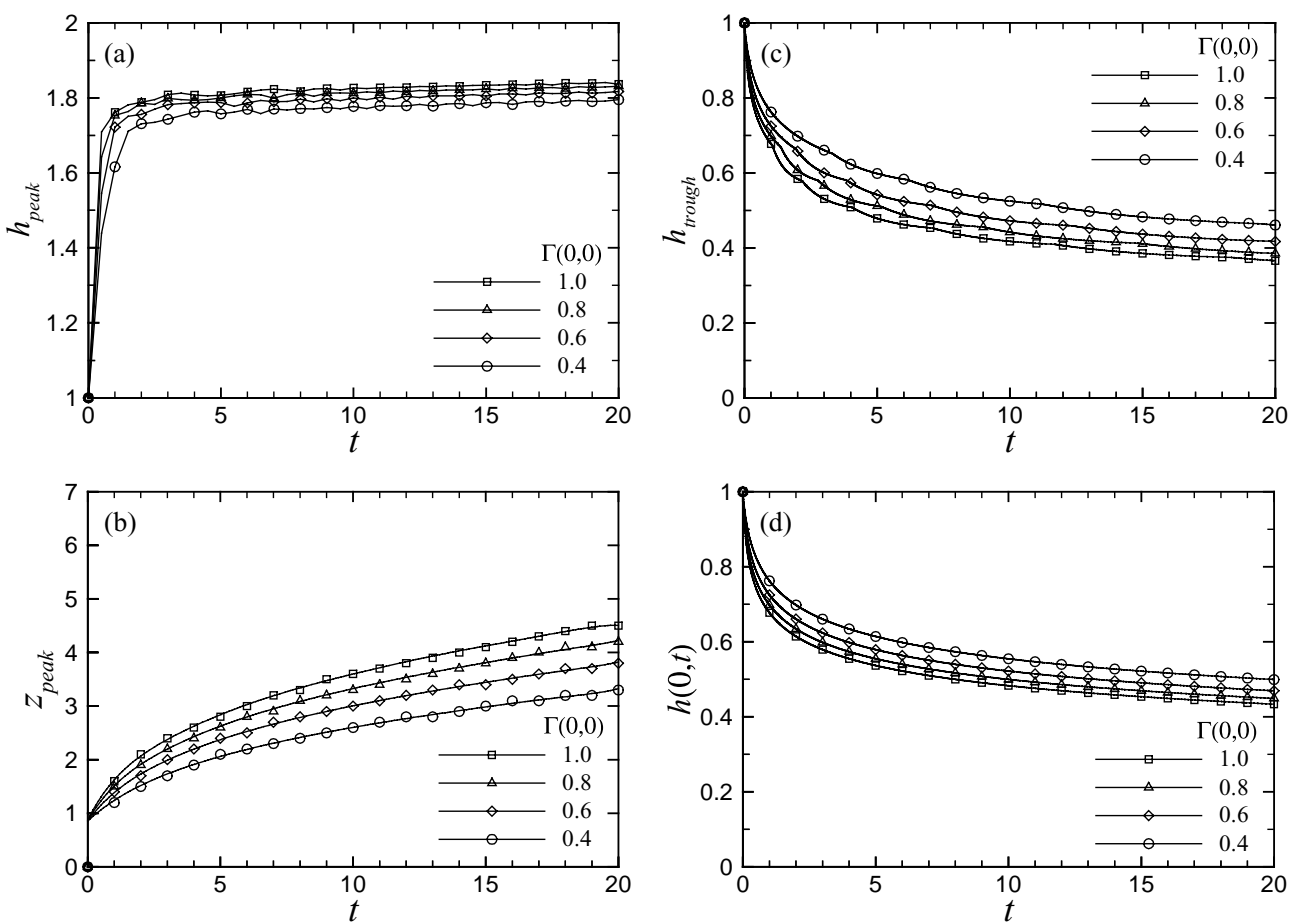

Fig. 7. Temporal evolutions of (a) the peak film thickness $h_{\text {peak }}$, (b) the axial coordinate of the peak $z_{\text {peak }}$, (c) the trough film thickness $h_{\text {trough }}$ and (d) the film thickness at entrance $h(0, t)$ for various exogenous surfactant concentrations $\Gamma_{\text {exo }}=1,0.8,0.6$ and 0.4 . The endogenous surfactant concentration is assumed to vanish, $\Gamma_{\text {end }}=0$, and the ratio of film thickness-to-airway radius $\varepsilon=0.01$.
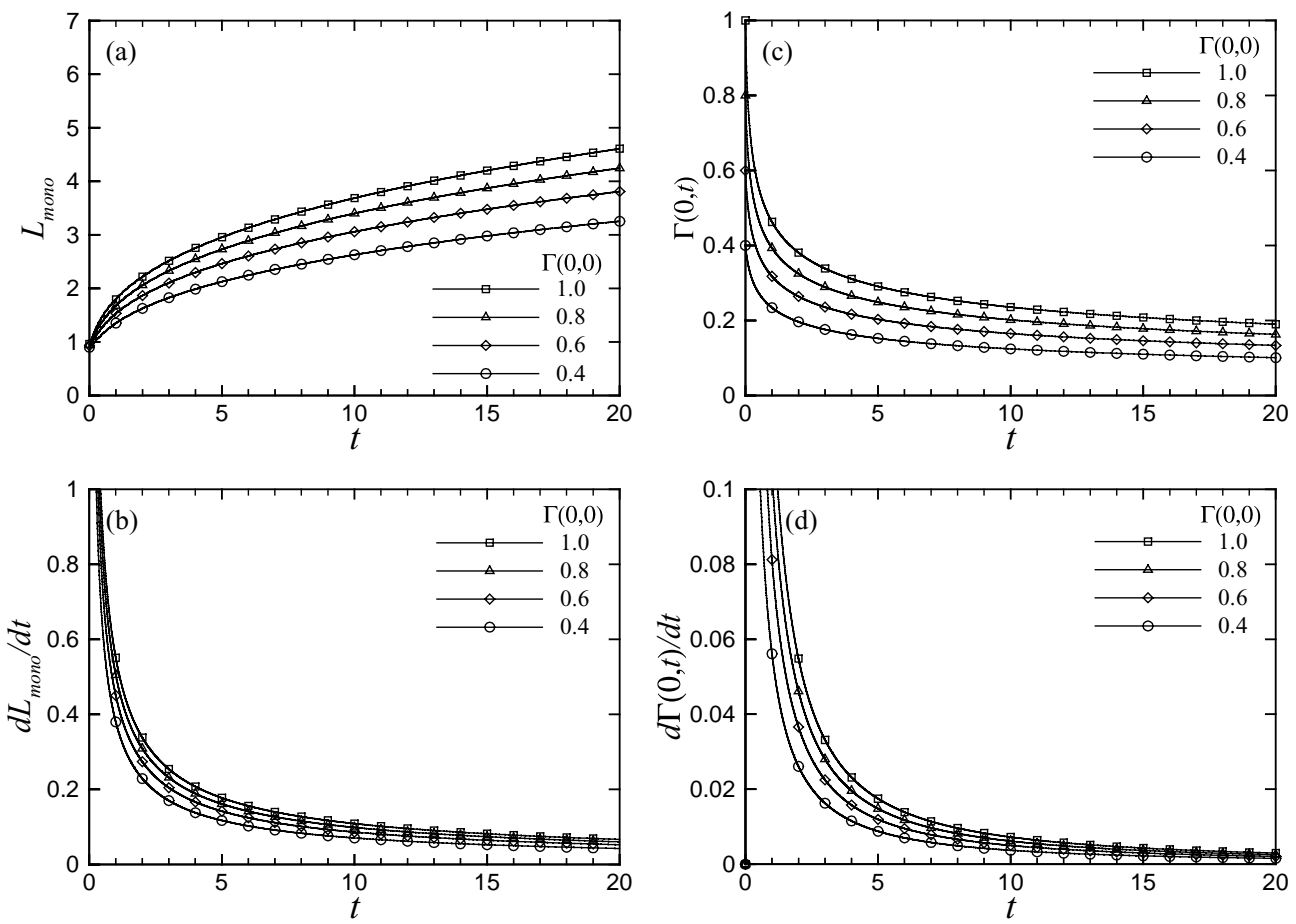

Fig. 8. Temporal evolutions of (a) the length of monolayer $L_{\text {mono, }}$ (b) the spreading velocity of surfactant $\mathrm{d} L_{\text {mono }} / \mathrm{d} t$, (c) the concentration at the entrance of airway $\Gamma(0, t)$ and (d) the dropping rate $\mathrm{d} \Gamma(0, t) / \mathrm{d} t$ for various exogenous surfactant concentrations $\Gamma_{\text {exo }}=1$, 0.8 , 0.6 and 0.4 . The endogenous surfactant concentration is assumed to vanish, $\Gamma_{\text {end }}=0$, and the ratio of film thickness-to-airway radius $\varepsilon=0.01$. 

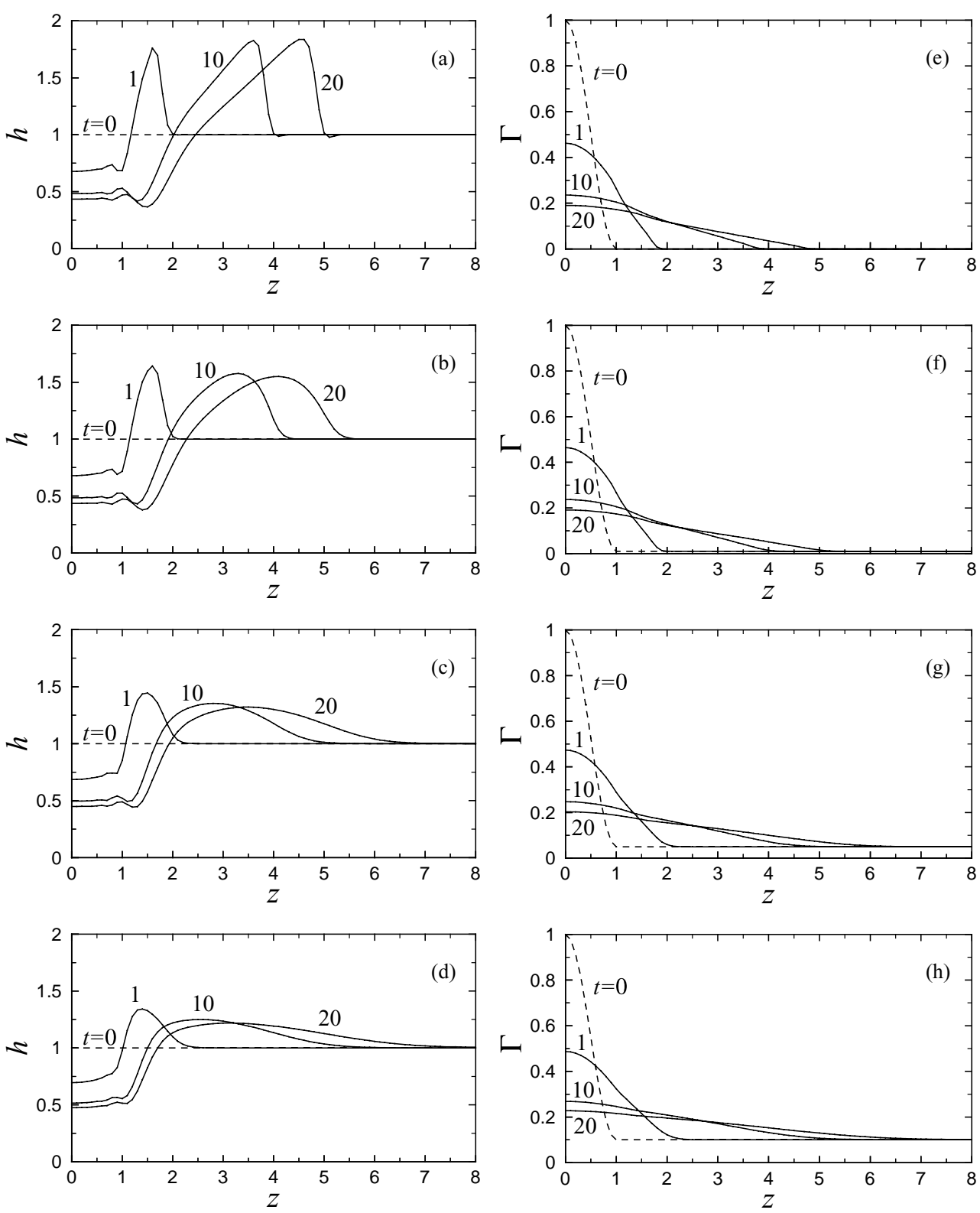

Fig. 9. Time varying profiles of film thickness $h(z, t)$ and surfactant concentration $\Gamma(z, t)$ for the initial endogenous concentration $\Gamma_{\text {end }}=0(\mathrm{a}, \mathrm{e}), 0.01$ $(\mathrm{b}, \mathrm{f}), 0.05(\mathrm{c}, \mathrm{g})$ and $0.1(\mathrm{~d}, \mathrm{~h})$. The initial concentration at $z=0$ is considered to be $\Gamma(0,0)=1$ for all cases and the ratio of film thickness-to-airway radius $\varepsilon=0.01$.

of the film disturbance and its propagation speed decrease upon increasing the endogenous concentration as depicted in Fig. 10. Nevertheless, contradictory effect of the endogenous concentration on advancing speed of the disturbance front is observed. Although the steepness and the peak height of the solitary disturbance are drastically reduced with the presence of resident surfactant, the extent of the disturbance, however, is moved forward. Such a prolonging effect also happens in surfactant concentration where the monolayer front seems to move faster for lining with pre-existing endogenous surfactant. Espinosa et al. [16] therefore conclude that the presence of an endogenous surfactant increases the spread- ing rate of the exogenous surfactant. Grotberg et al. [24], however, conclude differently by arguing that the higher frontal spreading rate is attributed to the compression of endogenous surfactant concentration in regions ahead of the advancing exogenous surfactant front. The exogenous surfactant, nevertheless, does not spread more efficiently with the presence of endogenous surfactant. The conclusion of Grotberg et al. [24] can also be reinforced by examining the decreasing trend of local concentration at $z=0$ (Fig. 11(c)), which reflects the spreading rate of instilled exogenous surfactant. For the case with endogenous surfactant $\Gamma_{\text {end }}=0.1$, the concentration level at $z=0$ is always higher than that of 



Fig. 10. Temporal evolutions of (a) the peak film thickness $h_{\text {peak }}$, (b) the axial coordinate of the peak $z_{\text {peak }}$, (c) the trough film thickness $h_{\text {trough }}$ and (d) the film thickness at entrance $h(0, t)$ for different values of initial endogenous concentration, $\Gamma_{\text {end }}=0,0.01,0.05$ and 0.1 . The initial concentration at $z=0, \Gamma(0,0)=1$, and the ratio of film thickness-to-airway radius $\varepsilon=0.01$
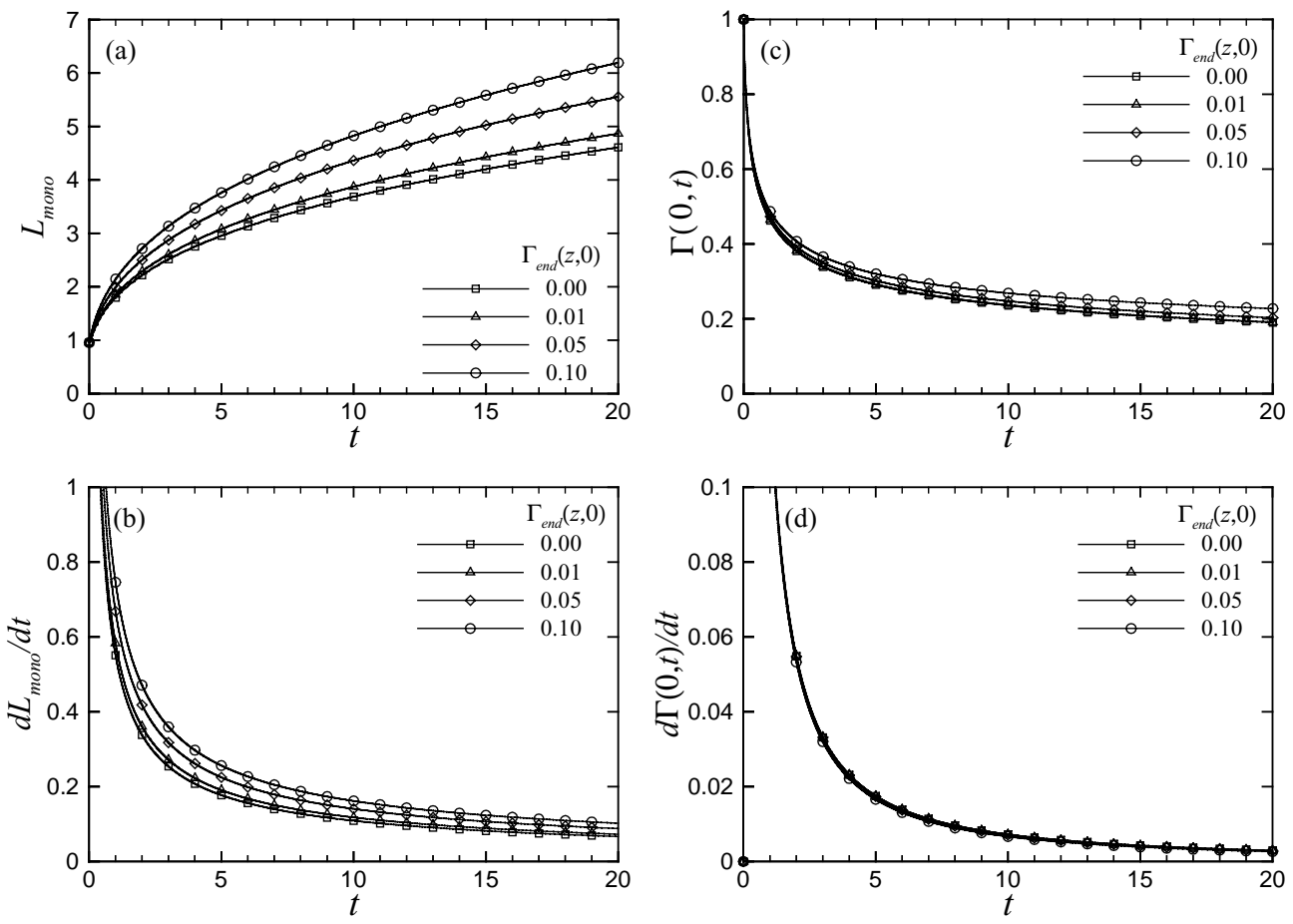

Fig. 11. Temporal evolutions of (a) the length of monolayer $L_{\text {mono }}$, (b) the spreading velocity of surfactant $\mathrm{d} L_{\text {mono }} / \mathrm{d} t$, (c) the concentration at the entrance of airway $\Gamma(0, t)$ and (d) the dropping rate $\mathrm{d} \Gamma(0, t) / \mathrm{d} t$ for different values of endogenous concentration, $\Gamma_{\text {end }}=0,0.01,0.05$ and 0.1 . The initial concentration at $z=0, \Gamma(0,0)=1$, and the ratio of film thickness-to-airway radius $\varepsilon=0.01$. 
$\Gamma_{\text {end }}=0$. No obvious change in the decreasing rate of $\Gamma(0$, $t$ ) is observed with various levels of endogenous surfactants (Fig. 11(d)).

\section{Concluding remarks}

We study the coupled transport of an axisymmetric bolus of insoluble surfactant instilled on a thin viscous film inside an airway of circular cross-section. The transport process is modeled by assuming a small film thickness relative to the airway radius, and the evolution of the viscous film is therefore governed by the balance between the viscous and Marangoni forces. Numerical simulation of the coupled model reveals that a propagating solitary shock forms on the film accompanying the spreading of surfactant and severe thinning of film occurs beneath the initial bolus of surfactant. Although the film depresses drastically, numerical results with extended simulation time demonstrate that such severe thinning of the film does not result in film rupture within a finite time. This is consistent with the conclusion of Jensen and Grotberg [13] for a planar lining that the film deformation subject to the viscous and Marangoni forces alone is never sufficient to drive the film to rupture.

Simulations for various ratios of film thickness-to-airway radius indicate that the non-dimensional properties of surfactant transport are nearly identical and exhibit spreading rate similarity. Increasing the airway radius (decreasing the circumferential curvature), however, tends to excite short-wavelength capillary disturbances in the region of depressed film. This suggests that the planar approximation employed in the previous studies would result in similar surfactant spreading rate as in the present axisymmetric case. The disturbed film height, nevertheless, would depend on the geometry (axisymmetric or planar) of the airway.

It is acknowledged that the low Reynolds number limit employed in the lubrication model is only valid for sufficiently large radius lining (i.e. low generation of airways) where the inertial effect is negligible in comparison with the viscous and Marangoni forces. Nevertheless, for airways higher than 10th generation, the radius decreases to less than $0.5 \mathrm{~mm}$ and the corresponding Reynolds number increases to $\mathrm{O}(1)$. This apparently violates the lubrication approximation of a thin film. Further studies, both numerical and theoretical, are necessary to determine how the spreading rate similarity is affected by such a non-linear effect.

The primary objective of the present ideal model is to clarify the underlying mechanisms that govern the transport of exogenous surfactant in an axisymmetric airway lining. The study highlights a number of issues that are relevant to SRT. The general results, however, do not imply that the efficiency of surfactant delivery in SRT is solely determined by the parameters considered. The effect of sorption attributed to surfactant solubility, the non-Newtonian properties of fluid lining and the geometry of more realistic pulmonary tree, among many others, all can influence the surfactant delivery into the lungs and the final distribution. These are issues under current investigation.

\section{Acknowledgements}

This work was supported by grants (90-2611-M-009-001 and 91-2611-M-008-002) from the National Science Council of Taiwan.

\section{References}

[1] M.E. Avery, J. Mead, Surface properties in relation to atelectasis and hyaline membrane disease, Am. J. Dis. Child. 97 (1959) 517 523 .

[2] T. Fujiwara, H. Maeta, S. Chida, T. Morita, Y. Watabe, T. Abe, Artificial surfactant therapy in hyaline-membrane disease, Lancet 1 (1980) 55-59.

[3] B. Robertson, H.W. Taeusch, Surfactant Therapy for Lung Disease, Marcel Dekker, New York, 1995.

[4] W.-T. Tsai, D.K.P. Yue, Effects of soluble and insoluble surfactant on laminar interactions of vortical flows with a free surface, J. Fluid Mech. 289 (1995) 315-349.

[5] B. Guyer, D.L. Hoyert, J.A. Martin, S.J. Ventura, M.F. MacDorman, D.M. Strobino, Annual summary of vital statistics-1998, Pediatrics 104 (1999) 1229-1246.

[6] A. Charon, H.W. Taeusch, C. Fitzgibbon, G.B. Smith, S.T. Treves, D.S. Phelps, Factor associated surfactant treatment response in infants with severe respiratory distress syndrome, Pediatrics 83 (1989) 348354.

[7] J.B. Grotberg, Respiratory fluid mechanics and transport processes, Annu. Rev. Fluid Mech. 3 (2001) 421-457.

[8] M.S. Borgas, J.B. Grotberg, Monolayer flow on a thin film, J. Fluid Mech. 193 (1988) 151-170.

[9] D.P. Gaver, J.B. Grotberg, The dynamics of a localized surfactant on a thin film, J. Fluid Mech. 213 (1990) 127-148.

[10] D.P. Gaver, J.B. Grotberg, Droplet spreading on a thin viscous film, J. Fluid Mech. 235 (1992) 399-414.

[11] D. Halpern, J.B. Grotberg, Dynamics and transport of a localized soluble surfactant on a thin film, J. Fluid Mech. 237 (1992) 111.

[12] D. Halpern, O.E. Jensen, J.B. Grotberg, A theoretical study of surfactant and liquid delivery into the lung, J. Appl. Physiol. 85 (1998) 333-352.

[13] O.E. Jensen, J.B. Grotberg, Insoluble surfactant spreading on a thin viscous film: shock evolution and film rupture, J. Fluid Mech. 240 (1992) 259-288.

[14] S.H. Davis, A.K. Liu, G.R. Sealy, Motion driven by surface tension gradients in a tube lining, J. Fluid Mech. 62 (1974) 737-751.

[15] F.F. Espinosa, Spreading of Surfactant in a Small Pulmonary Airway, Masters Thesis, Massachusetts Institute of Technology, 1991.

[16] F.F. Espinosa, A.H. Shapiro, J.J. Fredberg, R.D. Kamm, Spreading of exogenous surfactant in an airway, J. Appl. Physiol. 75 (1993) 2028-2039.

[17] J.L. Bull, L.K. Nelson, J.T. Walsh, M.R. Glucksberg, S. Schurch, J.B. Grotberg, Surfactant-spreading and surface-compression disturbance on a thin viscous film, J. Biomech. Eng. 121 (1999) 89-98.

[18] M.A. Krueger, Evaluation of physicochemical properties of a pulmonary surfactant analogue, Ph.D. Dissertation, Tulane University, 1999. 
[19] J. Morris, P. Ingenito, L. Mark, R.D. Kamm, M. Johnson, Dynamic behavior of lung surfactant, J. Biomech. Eng. 123 (2001) 106-113.

[20] J.S. Ultman, Gas transport in the conduction airways, in: Gas Mixing and Distribution in the Lung, Marcel Dekker, New York, 1985, Chapter 3, pp. 63-136.

[21] J.H. Widdicombe, Regulation of the depth and composition of airway surface liquid, J. Anat. 201 (2002) 313-318.
[22] E.K. Sakata, J.C. Berg, Surface diffusion in monolayers, Ind. Eng. Chem. Fundam. 8 (1969) 570-575.

[23] E.R. Weibel, Morphometry of the Human Lung, Academic Press, New York, 1963.

[24] J.B. Grotberg, D. Halpern, O.E. Jensen, Interaction of exogenous and endogenous surfactant: spreading-rate effects, J. Appl. Physiol. 78 (1995) 750-756. 